\title{
Microcalcifications segmentation using three edge detection techniques
}

\begin{abstract}
Edge detection has been widely used especially in medical image processing field. In this paper we are comparing Sobel, Prewitt and Laplacian of Gaussian (LoG) edge detection techniques in segmenting the boundary of microcalcifications. The edge detection must satisfy the breast phantom scoring criteria before the segmentation phase is carried out. Then, all of the edge detection techniques are implemented in the Enhanced Distance Active Contour (EDAC) model for the segmentation process. Results obtained from Area Under the Curve (AUC) of the Receiver Operating Characteristic (ROC) curve shows that the Prewitt edge detection has the highest value of AUC, followed by the Sobel and LoG which are 0.79, 0.72 and 0.71 respectively.
\end{abstract}

Keyword: Edge detection; Sobel; Prewitt; Laplacian of Gaussian; Segmentation; Mammogram 